\title{
Guideline: Überwachung und Management bei Überschreitung des Geburtstermins
}

\author{
Arbeitsgruppe «Terminüberschreitung»* der Kommission Qualitätssicherung der SGGG
}

\section{Präambel}

Aufbauend auf Evidence-based Medicine (EBM) sollen Guidelines eine Hilfeleistung und Orientierung für Diagnostik und Therapie an der individuellen Patientin erbringen. Die FMH legt Wert darauf, dass Guidelines insbesondere für die Ärzte in der Praxis, aber auch für den klinischen Bereich ihre Anwendung finden sollen. «Leitlinien» sind systematisch entwickelte Darstellungen und Empfehlungen mit dem Zweck, Arzt und Patientin bei der Entscheidung über zweckdienliche Massnahmen der Krankenversorgung (Prävention, Diagnostik, Therapie und Nachbehandlung) unter spezifischen klinischen Umständen zu unterstützen. Leitlinien stellen den Stand des Wissens über effektive und zweckdienliche Krankenversorgung zum Zeitpunkt der Veröffentlichung dar und wenden sich in erster Linie an den ärztlichen Bereich, erst sekundär an die Öffentlichkeit und die Versicherer.

Wegen der Fortschritte der wissenschaftlichen Erkenntnisse müssen Leitlinien periodisch überarbeitet werden. Die Entscheidung darüber, ob einer bestimmten Empfehlung gefolgt werden soll, muss vom Arzt unter Berücksichtigung der bei der individuellen Patientin vorliegenden Gegebenheiten und der verfügbaren Ressourcen entschieden werden. Im Einzelfall müssen somit die aktuelle Situation der Patientin, ihr Umfeld, ihre sozio-ökonomische Situation, die Komorbidität und Nebendiagnosen, aber auch ihre ethische und religiöse Haltung mitberücksichtigt werden. Damit ist ein entscheidender ärztlicher Freiraum gegeben, welcher es überhaupt ermöglicht, Leitlinien in die Praxis umzusetzen. «Therapeutische Freiheit» bedeutet, dass der Arzt sowohl die Kompetenz hat wie auch die Verantwortung dafür trägt, dass die von ihm an der Patientin vorgenommene Diagnostik bzw. durchgeführte Therapie dem aktuellen Stand der Wissenschaft entspricht - therapeutische Freiheit somit gleichermassen als Freiheit wie auch als Verpflichtung, das Richtige zu tun.

Das Erstellen und die laufende Aktualisierung von Leitlinien erfordern hohe Kompetenz, Beharrungsvermögen und zeitlichen Einsatz.
Vieles im Medizinberuf basierte schon immer auf Evidenz, neu sind eher die Methoden, Fortschritte und Kriterien, die eine Beurteilung der Validität von Studien und eine verständliche Darstellung ihrer Resultate ermöglichen. Eine gute Leitlinie zeigt unter anderem auf, für welche Situationen sie Handlungsempfehlungen anbietet, wie aktuell die Handlungsanweisung ist, welche Grade der wissenschaftlichen Sicherung für jede einzelne Aussage besteht und inwiefern ihre Befolgung der Patientin voraussichtlich einen Nutzen bringen wird. Gute Leitlinien werden den Diskurs zwischen Medizin und Recht (Decision Making Processes) verbessern.

\section{Experten}

Prof. Dr. med. P. Hohlfeld, Prof. Dr. med. G. Drack, Dr. med. P. Primavesi, Prof. Dr. med. H. Schneider, Dr. med. E. Vogel.

\section{Einführung}

\subsection{Relevanz des Themas}

Von Übertragung spricht man gemäss WHO und FIGO ab Vollendung von 42 Schwangerschaftswochen (ab $42+0$ Wochen), d.h. nach Überschreiten von 294 Tagen ab dem ersten Tag der letzten Monatsblutung. Im deutschen Sprachraum ist ausserdem für die Zeit von $40+1$ bis $41+6$ Schwangerschaftswochen der Begriff «Terminüberschreitung» üblich. Es scheint, dass ab dem Ende der 41. SSW ein Anstieg der neonatalen Morbidität beobachtet werden kann, also nach mehr als 287 Tagen ab dem ersten Tag der letzten Periode [1].

Die Inzidenz von Schwangerschaften mit längerer Dauer als 41 Wochen kann 27\% erreichen [2]. Die Inzidenz von Schwangerschaften mit mehr als 42 Wochen wird mit 4 bis $14 \%$, von denjenigen mit mehr als 43 Wochen mit 2 bis $7 \%$ angegeben [1]. Gegenwärtig scheint die Inzidenz der Übertragung abzunehmen, was zweifelsohne der genaueren Terminbestimmung durch die Ultraschalluntersuchung zuzuschreiben ist. 
Die Inzidenz der Übertragung hängt von mehreren Faktoren ab: der Definition (41 oder 42 Wochen), der untersuchten Population, der Praxis von Routinesonographien zur Terminbestimmung, der Frühgeburtenrate, den Anteilen von Geburtseinleitungen und von geplanten Schnittentbindungen.

Eine vorausgegangene Übertragung stellt einen Risikofaktor dar, da das Wiederholungsrisiko mit 30 bis $40 \%$ ermittelt wurde [3]. Darüber hinaus finden sich Übertragungen bei fetalen Fehlbildungen häufiger.

Bei Übertragung steigt das Risiko der perinatalen Mortalität [4, 5], und zwar antepartal, intrapartal wie auch postpartal. Ursachen dafür sind fetale Anomalien, intrauterine Infektionen und Asphyxie mit oder ohne mekonialem Fruchtwasser. Das Risiko der neonatalen Morbidität steigt ebenfalls an infolge Makrosomie, Schulterdystokie, Mekoniumaspiration, Intubation, Atemnotsyndrom, Persistenz des D. arteriosus, Pneumonie und zerebraler Schädigung oder gemessen am Anteil der Eintritte in die neonatologische Intensivstation [6-15].

Des weiteren führt die Übertragung zu einem höheren Anteil von Geburtseinleitungen, von fetalem Distress während der Geburt, von mekonialem Fruchtwasser und instrumentellen Entbindungen im Vergleich zu Termingeburten [16].

Bei Übertragung können sich weitere Risikofaktoren einstellen: Bluthochdruck oder Präeklampsie, Gestationsdiabetes, vorzeitige Lösung der Plazenta und Wachstumsretardierung.

\subsection{Ziel der Empfehlungen}

Diese Leitlinie zur Überwachung und zum Management bei Terminüberschreitung und Übertragung gründet auf publizierter wissenschaftlicher Evidenz und hat zum Ziel,

- die Überwachung und das Management bei echter Übertragung sicherzustellen;

- unnötige diagnostische und therapeutische Interventionen bei Schwangeren ohne eigentliche Übertragung zu vermeiden;

- die Mutter und ihren Partner in die Entscheidungsfindung zur Geburtsleitung miteinzubeziehen.

\subsection{Methode}

Diese Leitlinien wurden entsprechend den Vorgaben der Kommission Qualitätssicherung der SGGG erarbeitet.
- Die Kommission QS SGGG hat eine Arbeitsgruppe aus Ärzten gebildet, welche in Universitätskliniken, Regionalspitälern oder in einer Privatpraxis arbeiten. Auftrag dieser Gruppe war die Erarbeitung von Leitlinien für den Fall einer Übertragung.

- Die 5 Mitglieder der Arbeitsgruppe trafen sich im Verlaufe von zwei Jahren wiederholt und erarbeiteten so einen Konsens.

- Die bestehenden Guidelines und Studien wurden für die Jahre 1966 bis 1998 über eine Medline-Recherche gesucht und verarbeitet.

- Diese Leitlinien entsprechen den Praxishinweisen der Cochrane Library [17], US-amerikanischen [18] und kanadischen [19] Empfehlungen.

- Eine Überarbeitung der Leitlinien ist zwei Jahre nach der Publikation vorgesehen.

Grad der Evidenz (Canadian Task Force on the Periodic Health Examination [20])

Grad I: Die Evidenz ist aufgrund randomisierter kontrollierter Studien (oder Meta-Analysen) von genügendem Umfang derart, dass die Gefahr, dass sie falsch positive oder falsch negative Resultate beinhalten, gering ist.

Grad II: Die Evidenz basiert auf randomisierten kontrollierten Studien, welche jedoch zu klein sind, um ihnen Grad I zuzusprechen; sie können positive Trends, welche jedoch statistisch nicht signifikant sind, oder gar keine Trends zeigen. Sie sind mit einem hohen Risiko falsch negativer Resultate verbunden.

Grad III: Die Evidenz basiert auf nicht randomisierten Kontroll- oder Kohortenstudien, Fallserien, Fallkontrollstudien oder Querschnittstudien.

Grad IV: Die Evidenz basiert auf der Meinung angesehener Experten oder Expertengremien, wie sie in publizierten Konsenskonferenzen oder in Guidelines angegeben werden.

Grad V: Die Evidenz basiert auf der Meinung derjenigen Personen, welche diese Guidelines geschrieben oder aktualisiert haben, beruhend auf ihrer Erfahrung, ihrer Kenntnis der einschlägigen Literatur und der Diskussion mit ihren Fachkollegen.

\subsection{Definition und Zielgruppe}

Schwangerschaften von mehr als 40 Wochen Dauer (nach Bestätigung des Gestationsalters durch eine Ultraschalluntersuchung in der Frühgravidität). 


\section{Empfehlungen}

2.1 Bestimmung des Schwangerschaftsalters

- Die Betreuung einer Schwangerschaft bei Terminüberschreitung ist schwierig, wenn das Schwangerschaftsalter nicht sicher feststeht.

- In der Schweiz wird eine Ultraschalluntersuchung am Ende des ersten Schwangerschaftsdrittels empfohlen, wodurch in der überwiegenden Zahl der Fälle eine Unsicherheit bezüglich des Gestationsalters vermieden werden kann.

\subsection{Betreuung zwischen 39 und}

$40+6$ Schwangerschaftswochen

- Bei komplikationslosen Schwangerschaften besteht während dieser Periode kein Anlass zur Empfehlung einer Geburtseinleitung.

- Bei komplikationslosen Schwangerschaften besteht während dieser Periode auch kein Anlass zur Überwachung des Fetus.

- Bei Vorliegen von Risikofaktoren hingegen, wie z.B. Hypertonie, Diabetes, intrauterine Wachstumsretardierung, fetaler Grosswuchs, Polyhydramnion und Mehrlingen, sind eine engmaschige Überwachung oder die Geburtseinleitung zu erwägen.

\subsection{Betreuung mit 41 und 42 Schwanger- schaftswochen}

- Die Schwangeren sollten über Vorteile und Risiken der zwei denkbaren Haltungen informiert werden: Geburtseinleitung oder Abwarten. Die persönlichen Präferenzen der Schwangeren sind zu respektieren.

- Bei Erreichen eines Gestationsalters von 41 bis 42 Wochen nach komplikationslosem Verlauf sollte einer Schwangeren die Entbindung vorgeschlagen werden.

- Bei unreifer Zervix ist eine Reifungsinduktion mit einer der verfügbaren und anerkannten Methoden indiziert. Solange bei Mutter und Kind keine Probleme erkennbar sind, bedarf die Reifeinduktion keiner besonderen Eile.

- Bei reifer Zervix und ins Becken eingetretenem vorangehenden Teil können die Wehen ohne Schwierigkeiten durch Amniotomie, mit oder ohne Oxytozininfusion oder Verwendung von Prostaglandinpräparaten induziert werden. Die Entscheidung über die zu wählende Methode wird vom Arzt wie auch von der Schwangeren und den Kosten der einzelnen Verfahren abhängen.

\subsection{Abwartende Haltung}

- Entscheidungen zur Entbindung sind in Absprache mit der Mutter und ihrem Partner zu fällen. Einige Schwangere werden, trotz einer gewissen Senkung der Risiken (perinatale Mortalität und Morbidität, Schnittentbindung), keine Einleitung wünschen. In solchen Situationen ist die Überwachung des Feten zu veranlassen.

- Die Überwachung beinhaltet wenigstens eine zweimal wöchentlich vorzunehmende Schätzung der Fruchtwassermenge und ein CTG. Die Messung des grössten Fruchtwasserdepots scheint dabei einer Bestimmung des Fruchtwasserindexes überlegen zu sein $[17,21]$. Bei Vermutung eines Rückgangs der Fruchtwassermenge (grösstes Fruchtwasserdepot $<3 \mathrm{~cm}$ ) ist die Entbindung angezeigt. Es können weitere Formen zur Überwachung des Feten ergänzend beigezogen werden (biophysikalisches Profil nach Manning, Zählung der fetalen Bewegungen).

\subsection{Zusammenfassung der Empfehlungen}

- Schwangerschaften ohne besondere Risiken: Es gibt keine Evidenz zur Begründung einer Geburtseinleitung vor Vollendung von 41 SSW. Ebenso scheint eine Überwachung des Feten nicht notwendig zu sein.

- Bei Vollendung von 41 Schwangerschaftswochen (bei genau bestimmtem Schwangerschaftsalter): Der Schwangeren ist eine Geburtseinleitung anzubieten. Bei unreifer Zervix ist eine Zervixreifung vorzunehmen. Sofern Mutter und Kind bei guter Gesundheit sind, besteht für die Geburtseinleitung kein Grund zur Eile.

- Nach Vollendung von 41 Wochen, wenn die Schwangere keine Geburtseinleitung wünscht, ist eine engmaschige fetale Überwachung unerlässlich.

\section{Begründungen zu den Empfehlungen}

\subsection{Bestimmung des Schwangerschaftsalters}

Die exakte Bestimmung des Schwangerschaftsalters ist von wesentlicher Bedeutung. Damit sollen unnötige Interventionen bei Schwangeren ohne eigentliche Übertragung verhütet und gleichzeitig die notwendige Betreuung im Falle einer echten Übertragung sichergestellt werden.

Die zuverlässigste Methode zur Bestätigung oder Bestimmung des Schwangerschaftsalters ist die sonographische Untersuchung am Ende des ersten Trimenons [22]. Dies ist unerlässlich bei Unsicherheit über das Datum der letzten Men- 
struation, bei unregelmässigen Zyklen oder bei Eintritt der Schwangerschaft direkt nach Absetzen einer hormonalen Kontrazeption.

Studien mit einem Vergleich der Sonographie als Routineuntersuchung zur Sonographie auf Indikation zeigten bei einer Praxis mit routinemässiger Anwendung der Sonographie eine Verminderung der Rate an Geburtseinleitungen infolge vermeintlicher Übertragung $[17,23,24]$.

\subsection{Folgen einer Übertragung}

\section{Fruchtwassermenge}

Das Fruchtwasser weist bei 24 Schwangerschaftswochen sein maximales Volumen auf. Dieses bleibt bis 37 Schwangerschaftswochen in etwa konstant. Danach nimmt die Fruchtwassermenge fortlaufend bis zum Termin ab, und zwar um etwa $33 \%$ pro Woche. Diese Volumenreduktion geht mit einer Abnahme der Kindsbewegungen und einer Zunahme des Risikos von Nabelschnurkompressionen einher (CTG: variable Dezelerationen).

Plazenta

Die sonographischen Reifezeichen der Plazenta sind nicht Ausdruck eine funktionellen Veränderung der Plazenta. In Verbindung mit einem Oligohydramnion sind sie aber als Ausdruck der Übertragung aufzufassen.

\section{Geburtsgewicht}

Die Inzidenz von Makrosomien steigt um den Faktor 3 bis 7 an (etwa $40 \%$ der nach 42 Wochen geborenen Kinder wiegen über 4000 g), womit das Risiko von Schulterdystokien wächst.

\section{Dysmaturitätszeichen (Überreifesyndrom)}

Dieser Begriff bezieht sich auf die klinischen Zeichen an Neugeborenen, welche den Eindruck einer intrauterinen Mangelversorgung erwecken (Zeichen nach Clifford oder Runge). Die Zeichen finden sich bei 20 bis $40 \%$ der nach 42 Wochen geborenen Kinder.

\section{Perinatale Mortalität (PM)}

Der mit der Übertragung assoziierte Anstieg der perinatalen Mortalität betrifft vor allem subpartale und neonatale Todesfälle. Die gefährlichste Zeit fängt bei Geburtsbeginn an. Bei gleichzeitiger Wachstumsretardierung oder einer Pathologie auf seiten der Mutter ist die PM um den Faktor 4 bis 7 erhöht.

\section{Perinatale Morbidität}

Sie resultiert vorwiegend aus den Folgen einer Plazentainsuffizienz oder einer fetalen Makrosomie (akuter fetaler Distress, Mekoniumaspiration, geburtshilfliche Traumata, Hypoglykämie, Polyzythämie, Hypothermie).

\section{3 Überwachung des Fetus}

Es gibt zahlreiche Konzepte zur Überwachung des Fetus bei Terminüberschreitung. Eine umfassende Überwachung mit Kardiotokographie (CTG), Messung des sog. Fruchtwasserindexes, Prüfung der Atembewegungen, Bestimmung von Tonus und der fetalen Bewegungen (biophysikalisches Profil), scheint gegenüber einer alleinigen Überwachung mit CTG keine Vorteile aufzuweisen [17, 25].

Es bestehen nach wie vor einige Unsicherheiten bezüglich der Fähigkeit dieser Tests zur Aufdeckung einer fetalen Gefährdung wie auch zur Wirksamkeit der vorgeschlagenen Behandlungskonzepte im Falle positiver Tests [17].

Welcher Test auch immer gewählt wird, es gilt, sich die Möglichkeit einer raschen Änderung des fetalen Befindens vor Augen zu halten. Dies bedingt Kontrolluntersuchungen in kurzen Intervallen. Zudem können diese Tests falsch positive wie falsch negative Resultate ergeben.

Die Überwachung beinhaltet wenigstens eine zweimal wöchentlich vorzunehmende Schätzung der Fruchtwassermenge und ein CTG. Die Messung des grössten Fruchtwasserdepots scheint dabei einer Bestimmung des Fruchtwasserindexes überlegen zu sein $[17,21]$. Bei Vermutung eines Rückgangs der Fruchtwassermenge (grösstes Fruchtwasserdepot $<3 \mathrm{~cm}$ ) ist die Entbindung angezeigt. Es können weitere Formen zur Überwachung des Feten ergänzend beigezogen werden (biophysikalisches Profil nach Manning, Zählung der fetalen Bewegungen).

\subsection{Geburtseinleitung}

Die Einleitung der Geburt erscheint als eine einleuchtende Strategie bei Terminüberschreitung. Allerdings ist eine Einleitung bei unreifer Zervix nicht immer ein leichtes Unterfangen. In solchen Situationen scheint der Einsatz von Prostaglandinen zur Zervixreifung vor der eigentlichen Geburtseinleitung das Risiko einer Sektio zu vermindern [26, 27].

Einzelne Studien haben die Möglichkeit einer ambulant durchgeführten Geburtseinleitung geprüft [28-30]. Unserer Überzeugung nach gehört eine Geburtseinleitung ausserhalb von kontrollierten Studien in den stationär-klinischen Bereich. 


\subsection{Effektivität der Geburtseinleitung im Vergleich zur beobachtenden Haltung mit Überwachung des Feten}

Es liegen neunzehn randomisierte oder teilweise randomisierte Studien zum Vergleich einer Politik der Geburtseinleitung mit einer beobachtenden und den Fetus überwachenden Haltung vor [17].

In der Gruppe der expektativ betreuten Frauen kam es in 20 bis 36\% der Schwangerschaften zu einer Geburtseinleitung oder einer Schnittentbindung vor Geburtsbeginn [31]. Dies bedeutet, dass durch Abwarten nicht unbedingt ein spontaner Geburtsbeginn erreicht werden kann.

Es besteht kein sicherer Hinweis darauf, dass eine Politik der systematischen Geburtseinleitung die Wahrscheinlichkeit einer Schnittentbindung erhöht [17]. Laut gewissen Studien [26] ist die Rate von Sektiones bei Schwangeren mit einer Geburtseinleitung ab Vollendung von 41 Wochen oder danach signifikant kleiner als bei abwartend betreuten Schwangeren [26, 32]. Diese Differenz der Sektioraten liess sich sowohl bei Erst- wie bei Mehrgebärenden beobachten [26].

Durch die Weheninduktion mit Vollendung von 41 Wochen oder danach kann nach Angaben diverser Autoren die Zahl suspekter CTGs vermindert werden. Ebenso werden kleinere Raten an mekonialem Fruchtwasser oder an Makrosomien beobachtet [17].

Durch die Einleitung der Geburten mit Vollendung von 41 Wochen oder danach lässt sich im Vergleich zu einer abwartenden Haltung die fetale und neonatale Mortalität senken (bei Ausschluss kongenitaler oder schwerer Fehlbildungen; RR 0,23; CI $95 \%$ 0,06-0,90) [17]. Diese Reduktion ist vorwiegend auf die Verminderung des Risikos eines intrauterinen Todes zurückzuführen. Die Mehrzahl der in diesen Studien beobachteten Todesfälle war mit Asphyxie oder Mekoniumaspiration assoziiert.

Ausserdem liessen sich durch die Einführung einer Politik der Geburtseinleitung ab Vollendung von 41 Wochen oder danach im Vergleich zur abwartenden Haltung die Kosten der Betreuung senken [33].

\section{Zusammenfassung der geprüften Literatur}

Zusammenfassend ist festzuhalten, dass sich bei einer Übertragung das Risiko mütterlicher, fetaler oder neonataler Komplikationen erhöht. Perinatale Todesfälle sind häufig mit Asphyxieerscheinungen oder Mekoniumaspiration assoziiert. Es bestehen zwar zahlreiche Methoden zur
Überwachung des Feten bei Schwangerschaften am Termin, aber deren Effektivität ist noch wenig belegt.

Die randomisierten Studien zum Vergleich einer systematischen Geburtseinleitung ab Vollendung von 41 Wochen und einer abwartenden Haltung mit Überwachung des Feten zeigen, dass durch die Geburtseinleitung CTG-Anomalien, mekoniales Fruchtwasser, fetale Makrosomien und die perinatale Mortalität abnehmen. Die verfügbare Evidenz lässt den Schluss zu, dass eine Politik der systematischen Geburtseinleitung bei 41 bis 42 Wochen das Risiko einer Geburtsbeendigung durch Kaiserschnitt im Vergleich zu einer Politik des Abwartens nicht beeinflusst.

Schwangere sollen ab Vollendung von 41 Wochen in angemessener Weise aufgeklärt werden. Sie sollen über Risiken und Vorteile der verschiedenen Haltungen informiert werden. Die vorliegenden Studienresultate legen es nahe, mit der Schwangeren eine Geburtseinleitung zu besprechen. Man schätzt, dass auf 500 Geburtseinleitungen ein perinataler Todesfall verhindert wird [17].

Der Präsident der Kommission

Qualitätssicherung SGGG:

Prof. Dr. med. Urs Haller, Zürich:

Der Präsident der SGGG:

PD Dr. med. Patrick Hohlfeld, Lausanne:

\section{Literatur}

1 Feldman GB. Prospective risk of stillbirth. Obstet Gynecol 1992;79:547-53.

2 Beischer NA, Evans JH, Townsend L. Studies in prolonged pregnancy I. The incidence of prolonged pregnancy. Am J Obstet Gynecol 1969;103:476-82.

3 Bakketeig L, Bergsjo P. Post-term Pregnancy: Magnitude of the Problem. In: Chalmers I, Enkin M, Keirse MJNC (eds.). Effective Care in Pregnancy and Childbirth. Vol I. Oxford: Oxford University Press; 1989. p. 765-75.

4 Ingemarsson I, Kallen K. Stillbirths and rate of neonatal deaths in 76'761 postterm pregnancies in Sweden, 1982-1991: a register study. Acta Obstet Gynecol Scand 1997;76:658-62.

5 Hilder L, Costeloe K, Thilaganathan B. Prolonged pregnancy: evaluating gestation-specific risks of fetal and infant mortality. Br J Obstet Gynaecol 1998;105:169-73.

6 Crowley P. Post-term pregnancy: induction or surveillance? In: Chalmers I, Enkin M, Keirse MJNC (eds.). Effective Care in Pregnancy and Childbirth. Oxford: Oxford University Press; 1989. p. 776-91. 
7 Ballantyne JW, Browne FJ. The problems of fetal postmaturity and prolongation of pregnancy. J Obstet Gynaecol Brit Emp 1922;29:177-237.

8 McClure Browne JC. Postmaturity. Am J Obstet Gynecol 1963;85:573-82.

9 Naeye R. Causes of perinatal mortality excess in prolonged gestations. Am J Epidemiol 1978; 108:429-33.

10 Lucas WE, Anctil AO, Callagan DA. The problem of post-term pregnancy. Am J Obstet Gynecol 1965;91:241-50.

11 Clifford S. Postmaturity - with placental dysfunction: Clinical syndrome and pathologic findings: J Pediatr 1954;44:1-13

12 Curtis PD, Matthews TG, Clarke TA, Darling M, Crowley P, Griffin E, et al. Neonatal Seizures: the Dublin collaborative study. Arch Dis Child 1988;63:1065-8.

13 Saunders N, Paterson C. Effect of gestational age on obstetric performance: when is «term» over? Lancet 1991;338:1190-2.

14 McLean FH, Boyd ME, Usher RH, Kramer MS. Post-term infants: too big or too small? Am J Obstet Gynecol 1991;164:619-24.

15 Acker DB, Sachs BP, Friedman EA. Risk factors for shoulder dystocia. Obstet Gynecol 1985;66:762-8.

16 Shime J, Gare DJ, Andrews J, Bertrand M, Salgado J, Whillans G. Prolonged pregnancy: surveillance of the fetus and the neonate and the course of labor and delivery. Am J Obstet Gynecol 1984;148:547-52.

17 Crowley P. Interventions for preventing or improving the outcome of delivery at or beyond term (Cochrane Review). In: The Cochrane Library, Issue 1, 1999. Oxford: Update Software.

18 ACOG practice patterns. Management of postterm pregnancy. Number 6, October 1997. American College of Obstetricians and Gynecologists. Int J Gynaecol Obstet 1998;60:86-91.

19 Maternal-Fetal Medicine Committee, The Society of Obstetricians and Gynaecologists of Canada. Management of post-term pregnancy. Committee Opinion. Journal SOGC 1994;16:1581-6.

20 Clinical Practice Guidelines for the Care and Treatment of Breast Cancer. A Canadian Consensus document. Can Med Assoc J 1998: 158(3 Suppl).

21 Alfirevic Z, Luckas M, Walkinshaw SA, McFarlane M, Curran R. A randomised comparison between amniotic fluid index and maximum pool depth in the monitoring of post-term pregnancy. Br J Obstet Gynaecol 1997;104:207-11.
22 Gardosi J. Routine ultrasound is the method of choice for dating pregnancy. Br J Obstet Gynaecol 1998;105:933-6.

23 Neilson JP. Routine ultrasonography in early pregnancy. In: Chalmers I (ed.). Oxford database of perinatal trials. Version 1.3, Disk issue 7, Spring 1992, Record 3872.

24 Gardosi J, Vanner T, Francis A. Gestational age and induction of labour for prolonged pregnancy. Br J Obstet Gynaecol 1997;104:792-7.

25 Alfirevic Z, Walkinshaw SA. A randomised controlled trial of simple compared with complex antenatal fetal monitoring after 42 weeks of gestation. Br J Obstet Gynaecol 1995;102:638-43.

26 Hannah ME, Hannah WJ, Hellmann J, Hewson S, Milner R, Willan A, and the Canadian Multicenter Post-term Pregnancy Trial Group. Induction of labor as compared with serial antenatal monitoring in post-term pregnancy. A randomized controlled trial. N Engl J Med 1992;326:1587-92.

27 Keirse MJNC, Van Oppen ACA. Preparing the cervix for induction of labour. In: Chalmers I, Enkin M, Keirse MJNC (eds.). Effective care in pregnancy and childbirth. Oxford: Oxford University Press; 1989. p. 988-1056.

28 Sawai SK, O’Brien WF. Outpatient cervical ripening. Clin Obstet Gynecol 1995;38:301-9.

29 O’Brien JM, Mercer BM, Cleary NT, Sibai BM. Efficacy of outpatient induction with low-dose intravaginal prostaglandin E2: a randomized, double-blind, placebo-controlled trial. Am J Obstet Gynecol 1995;173:1855-9.

30 Ohel G, Rahav D, Rothbart H, Ruach M. Randomised trial of outpatient induction of labor with vaginal PGE2 at 40-41 weeks of gestation versus expectant management. Arch Gynecol Obstet 1996;258:109-12.

31 Crowley P. Induction of labour at 41+ weeks. In Chalmers I (ed.). Oxford Database of Perinatal Trials. Version 1.3, Disk Issue 7, Spring 1992. Record 4142.

32 Dyson D, Miller PD, Armstrong MA. Management of prolonged pregnancy: induction of labor versus antepartum testing. Am J Gynecol Obstet 1987; 156:928-34.

33 Goeree R, Hannah M, Hewson S. Cost-effectiveness of induction of labour versus serial antenatal monitoring in the Canadian Multicentre Postterm Pregnancy Trial. Can Med Assoc J 1995; 152:1445-50. 